\title{
Intracisternal Schwannoma of the Spinal Accessory Nerve: A Case Report
}

\author{
Tae-Young Jung, M.D., ${ }^{1}$ Shin Jung, M.D., Ph.D., ${ }^{1}$ In-Young Kim, M.D., ${ }^{1}$ \\ and Sam-Suk Kang, M.D.'
}

We report the details of a patient with an intracisternal schwannoma that developed from the spinal accessory nerve. The patient, a 70-year-old women, presented with a 5-year history of intermittent headache and neck pain. A $3.2 \times 2.5 \mathrm{~cm}$ partially cystic mass was found in the right cervicomedullary cistern. It was removed through a far-lateral inferior suboccipital craniotomy using image-guidance. The tumor arose from one rootlet of the right accessory nerve and histological examination confirmed the diagnosis of a schwannoma. Removal of the schwannoma did not result in a significant neurological deficit.

KEYWORDS: Intracisternal, schwannoma, spinal accessory nerve

\begin{abstract}
Accessory nerve schwannomas are extremely rare and can be characterized by their location as either intrajugular or intracisternal, most being intrajugular. ${ }^{1}$ Preoperative clinical diagnosis is almost impossible because of the difficulty in determining the nature and origin of the mass due to crowding of the lower cranial nerves. ${ }^{2-4}$

We report on a case of intracisternal schwannoma that arose from the spinal accessory nerve and discuss the postoperative implications of such a tumor.
\end{abstract}

\section{CASE REPORT}

A 70-year-old woman presented with an intermittent headache and neck pain that had troubled her for about 5 years. On examination there were no neurological deficits. Her neck movements were normal for her age and there was no wasting or asymmetry of her neck musculature, in particular the trapezius and sternocleidomastoid muscles. A computed tomography (CT) scan showed that she had a mass of $3.2 \times 2.5 \mathrm{~cm}$ in the right side of her

Skull Base 2006;16:175-180. Copyright (C) 2006 by Thieme Medical Publishers, Inc., 333 Seventh Avenue, New York, NY 10001, USA. Tel: +1(212) 584-4662.

Received: August 9, 2005. Accepted after revision: August 31, 2005. Published online: May 17, 2006.

DOI 10.1055/s-2006-939678. ISSN 1531-5010. 
A
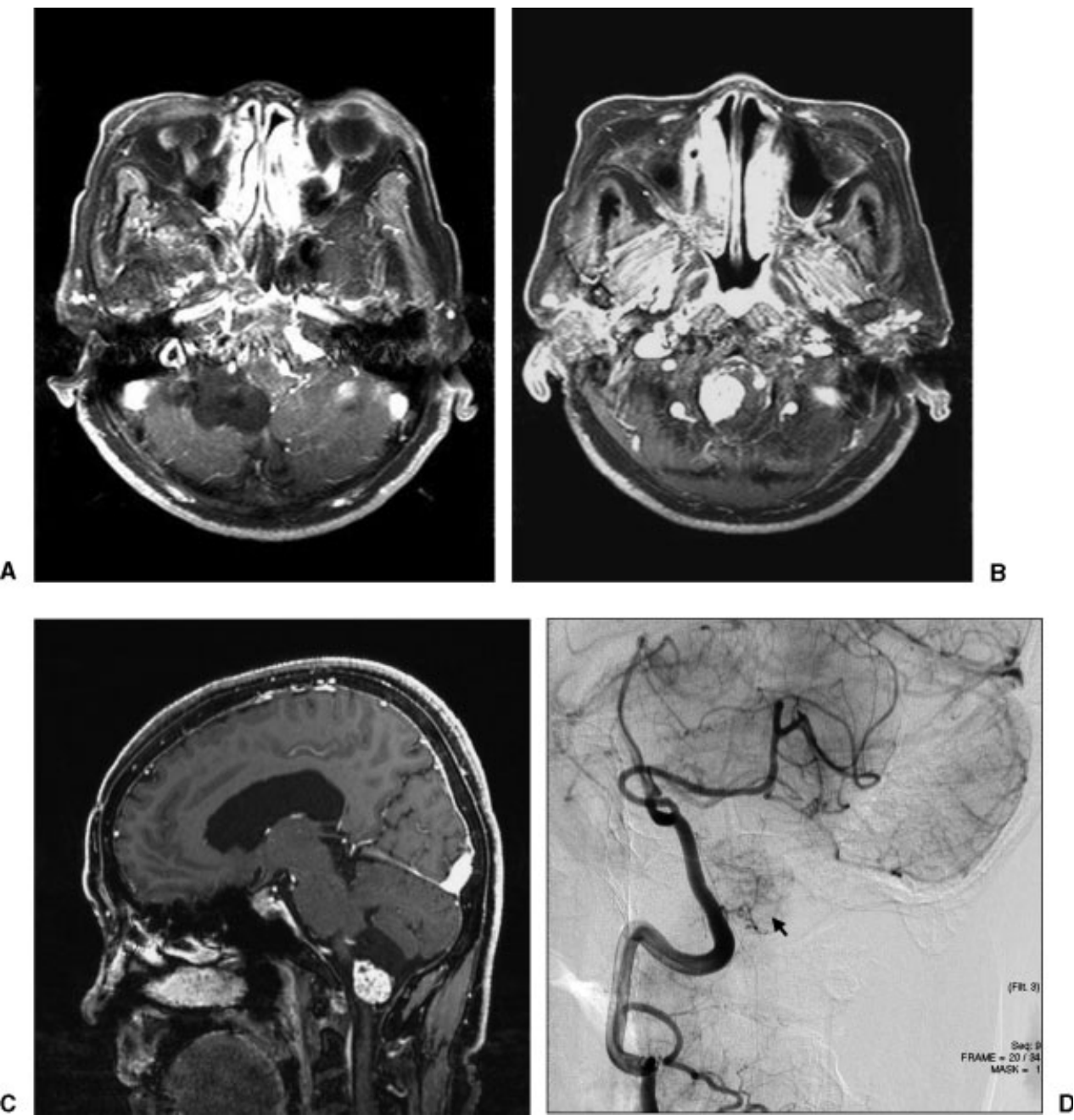

Figure 1 (A) Axial T1-weighted image shows a multilobulated well-defined lesion in the right cervicomedullary cistern. (B) Axial gadolinium T1-weighted image shows uniform enhancement of the mass showing severe compression of the medulla. (C) Sagittal gadolinium T1-weighted image shows severe impingement of the craniocervical brainstem. (D) Vertebral angiogram illustrates the mild hypervascularity of the tumor which was supplied by a small branch of the extracranial portion of the right vertebral artery (arrow).

posterior fossa that had both cystic and solid components. Magnetic resonance imaging (MRI) showed that the mass was well defined and in the right cervicomedullary cistern. It displaced the medulla and was multilobular. The solid components of the mass enhanced homogeneously with gadolinium while the cystic areas did not (Figs. 1A,B,C). A provisional diagnosis of schwannoma or cystic meningioma was made. The patient underwent cerebral angiography which showed that the tumor was mildly hypervascular and supplied by a small branch of the extracranial portion of the right vertebral artery (Fig. 1D). It was decided to undertake preoperative embolization because the feeding artery was easy to access and this would facilitate surgical resection. Transarterial embolization of the feeding vessel was achieved with polyvinyl alcohol particles and Gelfoam ${ }^{\mathrm{TM}}$. This resulted in the complete devascularization of the tumor. It was this point that the diagnosis of schwannoma became more clear as the tumor was not as vascular as had previously been thought.

An image-guided, far-lateral, inferior suboccipital craniotomy was performed. As the cisterna 
A
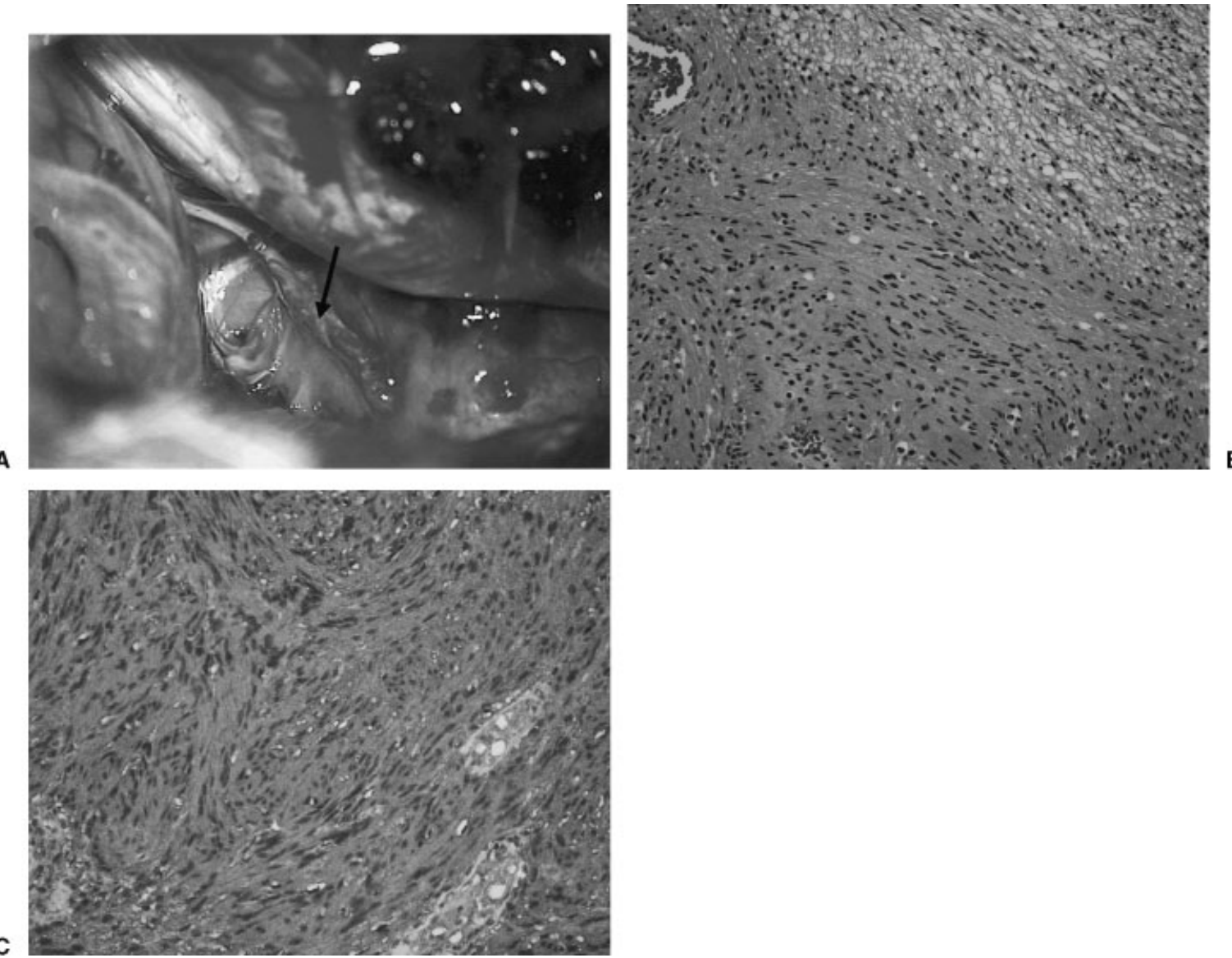

Figure 2 (A) The spinal accessory rootlet near the spinal cord was displaced and entered the tumor (arrow indicates spinal accessory rootlet). (B) Hypercellular Antoni A areas within the tumor (original magnification $\times 200$ ). (C) The tumor cells were immunopositive for S-100 marker (original magnification $\times 200$ ).

magna was opened a solid, capsulated, and finely vascularized tumor became visible. There were obvious cystic areas. The tumor displaced the medulla medially and the spinal accessory rootlet near to the spinal cord was fanned out before entering the tumor (Fig. 2A). The rootlet was cut on the proximal and distal portion of the mass and a total macroscopic resection was achieved. The vagal and glossopharyngeal nerves were preserved. At completion of the resection it became clear that the tumor had developed from one small branch of the right accessory nerve.

Histopathological examination showed an encapsulated mass with hypercellular (Antoni Type A pattern) and hypocellular (Antoni Type B pattern) areas (Fig. 2B). The hypercellular areas were com- posed of compact spindle cells with twisted nuclei arranged in short bundles of interlacing fascicles and showed nuclear palisading and whorls of cells (Verocay bodies). The hypocellular areas were composed of wavy spindle cells and oval cells in a loose stroma. No tumor cells were seen in the walls of the cysts. The tumor cells were immunopositive for S-100 and immunonegative for GFAP (glial fibrillary acidic protein) and EMA (epithelial membrane antigen) (Fig. 2C). The mass was confirmed to be a schwannoma.

The patient made an uneventful recovery during which she experienced some mild neck pain. There were no cranial nerve deficits and she did not develop any shoulder weakness or impairment of neck movement. 


\section{DISCUSSION}

The majority of schwannomas found in the cerebellopontine angle arise from the vestibulocochlear nerve. Schwannomas of the lower cranial nerves are rare and when present usually arise in the jugular foramen. In a series of 238 cerebellopontine angle schwannomas reported by Pluchino and associates, 12 originated in the jugular foramen. ${ }^{5}$ Schwannomas in jugular foramen neurinomas have most commonly developed on the glossopharyngeal or vagal nerves. The accessory nerve is the least common site of origin. ${ }^{6,7}$ Sometimes it is extremely difficult to determine at the time of surgery precisely on which root the tumor has developed. In a previous study, these tumors presented with classical jugular foramen symptoms from which it too was impossible to incriminate one nerve or another. ${ }^{4}$

Julow presented two cases of accessory nerve schwannomas and suggested that they could be divided into two groups, namely, intrajugular schwannomas that grow into the cisterna magna and intracisternal schwannomas that grow into the jugular foramen. ${ }^{1}$ The clinical symptoms of intrajugular schwannomas consist of various combinations of 5 th to 12 th cranial nerve palsies, cerebellar dysfunction, and myelopathy, depending on the degree of their extension. ${ }^{4}$ Patients with intrajugular schwannomas usually present with a jugular foramen syndrome because the tumor is confined entirely within the foramen. Radiological diagnosis of jugular foramen tumors using CT or conventional $\mathrm{x}$-rays is often difficult. ${ }^{8}$ In some cases enlargement of the jugular foramen is evident on CT and this is a valuable diagnostic feature. MR has made diagnosis much easier even though it is still not possible to identify the nerve of origin. ${ }^{9,10}$

Patients with intracisternal schwannomas usually have an accessory nerve palsy, cerebellar signs, and/or myelopathy. Deficits of glossopharyngeal or vagal function have not been reported. ${ }^{1}$ Our patient had an intracisternal tumor that had caused long-standing neck stiffness possibly due to accessory nerve irritation. The precise diagnosis of her tumor was difficult to make prior to surgery. ${ }^{11}$
The MR appearances of these tumors usually have regular contours and a round or oval shape, lack edema, and enhance homogenously with contrast. Cystic degeneration is generally seen in large tumors and this may alter the otherwise uniform texture of the mass. ${ }^{12}$ These tumors are generally found against the posterior border of the foramen occipitalis, close to the midline. ${ }^{1,13}$

Preoperatively, the presence of specific accessory nerve signs may depend on which part of the nerve is infiltrated and how much the tumor has expanded into the nerve trunk. ${ }^{1}$ Postoperative accessory nerve deficits also seem to depend on the degree of damage to the nerve. In the case reported by Caputi and colleagues, dissection was performed along the arachnoid interface, exposing the tumor attachment to the spinal accessory nerve, from which it was removed en bloc with no apparent damage caused to the nerve. ${ }^{13}$ Their patient recovered completely without any impairment of neck movement. However, in another case in which the fibers of the accessory nerve entered the tumor and were coagulated and cut, the patient experienced shoulder muscle weakness and impairment of neck motion postoperatively. ${ }^{14}$ So, in cases where the mass originates from a spinal accessory rootlet, the damage to the rootlet does not lead to the development of specific accessory nerve signs. ${ }^{1}$ In our case, a spinal accessory rootlet near the spinal cord was the place of origin of the tumor and resulted in a lack of postoperative symptoms and signs.

Total removal of these tumors is recommended as recurrence is probably unavoidable if removal is incomplete. ${ }^{14}$

\section{REFERENCES}

1. Julow J. Neurinoma of the spinal accessory nerve: report of two cases. Acta Neurochir (Wien) 1983;69:219-224

2. Iwasaki K, Kondo A. Accessory nerve neurinoma manifesting with typical jugular foramen syndrome. Neurosurgery 1991;29:455-459

3. Pluchino F, Crivelli G, Vaghi MA. Intracranial neurinomas of the jugular foramen. Acta Neurochir (Wien) 1975; 31:201-221 
4. Shiroyama $\mathrm{Y}$, Inoue $\mathrm{S}$, Tsuha $\mathrm{M}$, Abiko $\mathrm{S}$, Aoki $\mathrm{H}$. Intracranial neurinomas of the jugular foramen and the hypoglossal canal [in Japanese]. No Shinkei Geka 1988; 16:313-319

5. Pluchino F, Crivelli G, Vaghi MA. Intracranial neurinomas of the nerves of the jugular foramen. Acta Neurochir (Wien) 1975;31:201-221

6. Fink LH, Early CB, Bryan RN. Glossopharyngeal schwannomas. Surg Neurol 1978;9:239-245

7. Hakuba A, Hashi K, Fujitani K, et al. Jugular foramen neurinomas. Surg Neurol 1979;11:83-94

8. Solymosi L, Wassmann H, Bonse R. Diagnosis of neurinoma in the region of the jugular foramen. A case report. Neurosurg Rev 1987;10:41-46

9. Sugawara A, Kowada M, Nishino K, Sakamoto T. Jugular foramen neurinoma-report of a case. No Shinkei Geka 1981;9:829-833

10. Suzuki S, Yamaguchi K, Sakai H, Tanji H, Higa K. Intracranial neurinoma of jugular foramen-report of a case and reference, its clinical manifestations (author's trans) [in Japanese]. No Shinkei Geka 1977;5:379384

11. Caputi F, de Sanctis S, Gazzeri G, Gazzeri R. Neuroma of the spinal accessory nerve disclosed by a subarachnoid hemorrhage: case report. Neurosurgery 1997;41:946950

12. Matsushima T, Hasuo K, Yasumori K, et al. Magnetic resonance imaging of jugular foramen neurinomas. Acta Neurochir (Wien) 1989;96:83-87

13. Uchino A, Hasuo K, Fukui M, et al. Computed tomography of jugular foramen neurinomas. Report of four cases. Neurol Med Chir (Tokyo) 1987;27:628632

14. Lanotte M, Massaro F, Scienza R, Faccani G. Intracisternal schwannoma of the spinal accessory nerve presenting as a normal pressure hydrocephalus syndrome. Case report and review of the literature. Neurosurg Rev 1994;17:225-227 